\title{
Enjeux identitaires et sociétaires : à la recherche d'une adéquation entre discours et attentes dans la construction des représentations sociales du sénior
}

\author{
Anne-Marie Cotton, Doctorante MICA (Bordeaux \\ 3), maître de conférences à la Haute Ecole \\ Artevelde à Gand (Belgique), \\ am.cotton@arteveldehs.be \\ Agnès d'Arripe, Docteur, maître de conférences, \\ Université Catholique de Lille, \\ agnes.darripe@univ-catholille.fr
}




\section{Résumé}

L'étude de cas d'un système de téléalarme permet d'aborder l'adéquation entre les attentes du sénior actif et les discours du communicant jouant un rôle dans la construction des représentations sociales autour du sénior. Dans une société axée sur la productivité, où l'injonction d'autonomie est centrale, le sénior se voit soumis à une norme du «bien vieillir ». En resituant le sénior dans son histoire personnelle et collective, nous étudions le discours du communicant de la start-up belge Zembro à son égard et les constructions symboliques engendrées ou mobilisées. Tantôt représenté positivement dans une "horizontalité des pairs", retraité aux multiples activités, tantôt négativement dans une "verticalité des pères", vieillard dépendant, comment s'adresser à la fois au sénior et à ses proches qui participent à son autonomie conçue comme solidaire ou relationnelle ? Comment intégrer les proches souvent prescripteurs sans faire du sénior un «individu collectif ») sans réelle prise sur ce qui est important pour lui? Une approche thématique reposant sur l'adéquation « discours-cible » a permis de définir la place qu'un système de téléalarme prend ou pourrait prendre dans la vie d'un sénior et sa position par rapport au discours d'une entreprise.

Mots-clés : Sénior, bracelet intelligent, représentations sociales, autonomie relationnelle, objet technologique, TIC

\footnotetext{
Abstract

The case study of a remote alarm system addresses the adequacy between the expectations of the active senior and the communication director's discourse having a role in the construction of social representations around the senior. In a productivitydriven society focusing on autonomy, the senior is strongly invited to adopt the "age well" standards. Contextualising the senior in his personal and collective history, we study this discourse of the Belgian start-up Zembro and the symbolic constructions it engenders or mobilizes. Sometimes represented positively in a "peer horizontality", the retired with multiple activities, sometimes negatively in a "verticality of the fathers", the dependent old man, how to address both the senior and his relatives who participate in its autonomy conceived as solidary or relational? How to integrate the close relatives often prescribers without making the senior a "collective individual" that would no longer have real hold on what is important for him? A thematic approach of this case study based on the "discourse-public" adequacy, allowed us to define the place a remote alarm system takes or could take in the senior's life and his position toward the discourse emanating from a profit organisation.

Keywords: Sénior, smart wristband, social representations, relational autonomy, technological object, ICT
} 
Si l'âge de 60 ans s'est imposé comme entrée dans la vieillesse au 18ième siècle avec le développement des statistiques, c'est en 1850 que le socialiste français Pierre Leroux introduit - en le stigmatisant - le problème soulevé par cette nouvelle catégorie sociale «à charge » en posant la question «Qu'en faut-il faire ? » (Hummel, 2002 , p. 68). Une question d'autant plus actuelle que l'espérance de vie ne cesse d'augmenter tout comme l'accroissement de l'indice de séniorité, composé par la part des plus de 80 ans, soit un vieillissement dans le vieillissement (Bawin-Legros et Casman, 2001). Avec la promesse de maintenir une autonomie comme antidote à la dépendance et à la relégation (Amyot, 2014) et celle de faire reculer le spectre de la maladie d'Alzheimer et de son cortège de troubles identitaires, cette catégorie sociale que nous identifions comme le «sénior " 1 doit se conformer à la norme du bien vieillir. Cette norme implique une injonction d'autonomie radicale (Amyot, 2012). Le vieillissement réussi est celui où on garde un bon physique, où on est socialement engagé, autrement dit où on ne vieillit pas (Balard, 2013). Ce diktat du « bien vieillir » (Amyot, 2012) est diffusé dans la presse, via les brochures éditées par le monde de l'action sociale ou gérontologique, par la communication autour d'objets ou de services destinés aux séniors...Nous présenterons ce que la littérature nous enseigne sur le « comment vieillir » avant d'aborder la cible du sénior et ses enjeux. Nous nous pencherons sur le rôle que peut ou doit jouer le communicant dans la construction des représentations sociales du sénior. Partant de l'impact du vieillissement sur la représentation identitaire, quelle typologie en extraire, quels choix stratégiques se présentent au communicant et quelle posture adopte-t-il ? Nous tenterons de répondre à ces questions en réalisant une étude de cas portant sur la communication de la startup belge, Zembro, qui a développé en 2015 un bracelet-alarme pour le segment des « séniors actifs ». Zembro nous a semblé un cas intéressant à analyser pour plusieurs raisons. Tout d'abord, il s'agit d'une start-up : sans référents communicationnels historiques, la marque peut s'approprier un territoire communicationnel différencié. Dans un secteur dominé par des dispositifs para publics ou associatifs, Zembro se positionne comme une entreprise à but lucratif. Ensuite, primée par ses pairs dans le domaine du marketing pour son approche innovante, Zembro a développé un système d'alarme personnelle connectée pour une génération a priori non connectée. Enfin il s'agit d'un objet qui touche à l'intégrité de la personne, qui a un impact sur sa vie privée. La construction de sens autour du produit implique de tenir compte du cycle de vie de la cible plus que de celui du produit. Elle sous-entend également la question du public à qui le communicant va s'adresser : la personne âgée elle-même, ses proches, les professionnels du vieillissement? Au-delà de la communication instrumentale, par son choix stratégique, le communicant va mobiliser ou induire des

1 Nous reviendrons ultérieurement sur cette catégorie sociale du sénior 
représentations et contribuer à la construction d'une image de la personne âgée en perte d'autonomie au sein de la société.

\section{La méthodologie}

\subsection{La définition des thèmes}

Nous avons abordé notre problématique comme une étude de cas dont la méthodologie consiste « à rapporter une situation réelle prise dans son contexte, et à l'analyser pour voir comment se manifestent et évoluent les phénomènes auxquels le chercheur s'intéresse » (Mucchielli, 2009, p.91). Notre démarche étant inductive, nous avons exclu les études de cas multiples qui s'inscrivent «dans une perspective de comparaison de résultats entre plusieurs situations afin d'en dégager les récurrences constitutives de l'élaboration graduelle d'une théorie » (Duvernay, 2011).

Nous avons défini une approche thématique reposant sur l'adéquation « discourscible » en nous basant sur une phase exploratoire composée d'une revue de la littérature, d'une interview du directeur de marketing et de communication de Zembro et d'une analyse thématique du site Internet et de la brochure de Zembro, ainsi que des sites Internet de 15 concurrents ( 3 belges, 3 canadiens et 9 français).

Nous avons pu identifier 6 binômes : Autonomie / déprise ; Liberté / dépendance ; Engagement social / désintérêt; Pouvoir / perte de contrôle; Acceptation / culpabilisation; Individu en contrôle de soi à domicile / individu incapacitaire institutionnalisé.

Le tableau ci-dessous illustre cette façon de travailler. 
133 Enjeux identitaires dans la construction des représentations

\begin{tabular}{|c|c|c|c|c|c|c|c|c|c|c|c|}
\hline 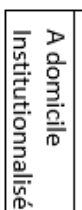 & & 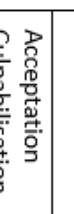 & 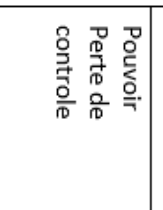 & & 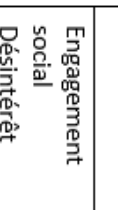 & 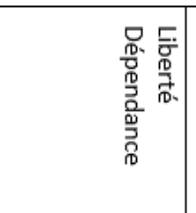 & 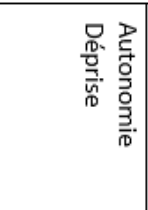 & 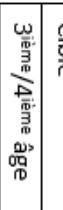 & & 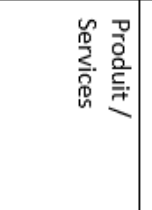 & \\
\hline 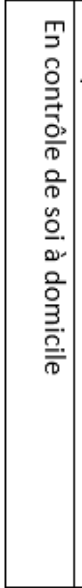 & 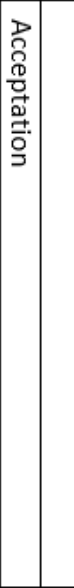 & 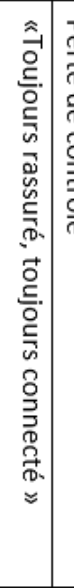 & 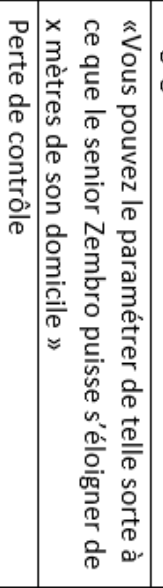 & & 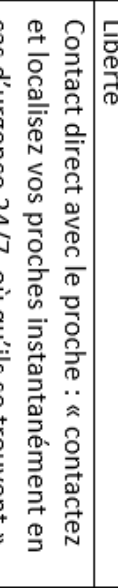 & 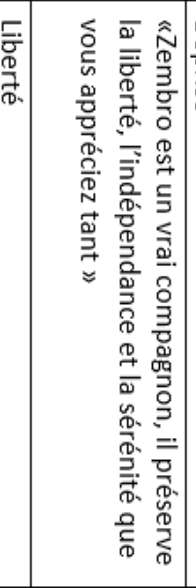 & 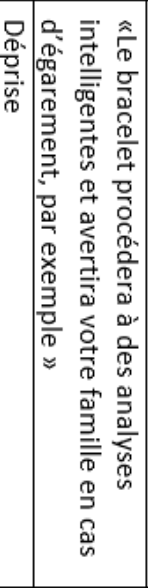 & 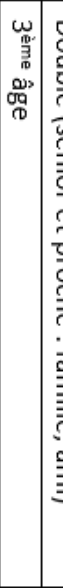 & & 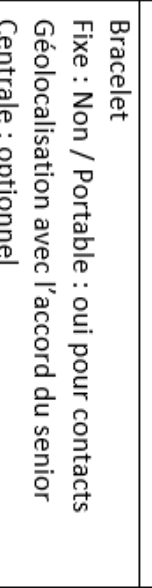 & \\
\hline 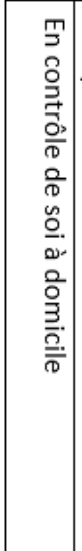 & 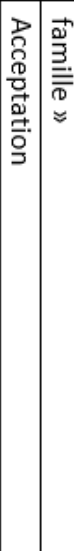 & 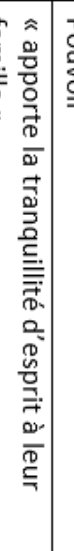 & 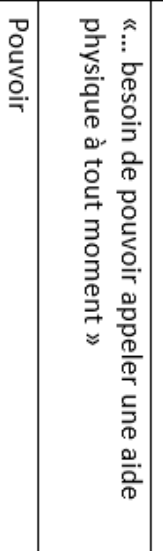 & 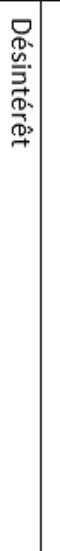 & 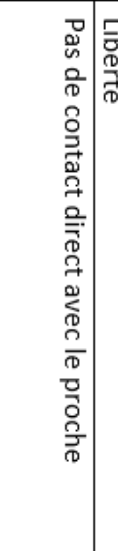 & 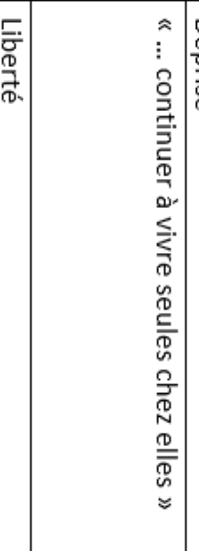 & 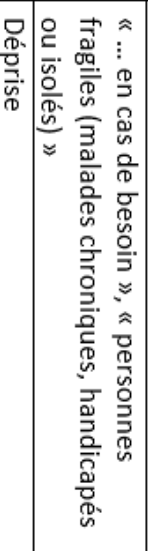 & 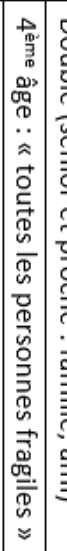 & 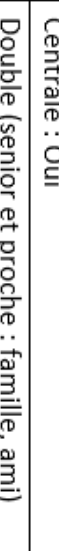 & 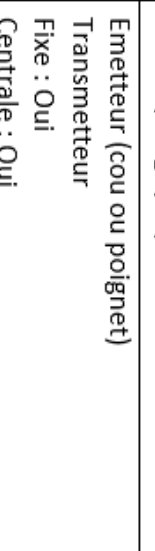 & \\
\hline $\begin{array}{l}\frac{\partial}{\partial} \\
\overline{0} \\
\underline{0} \\
\overline{0} \\
\underline{o} \\
\underline{\alpha} . \\
\end{array}$ & 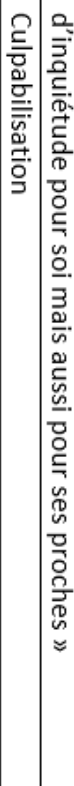 & 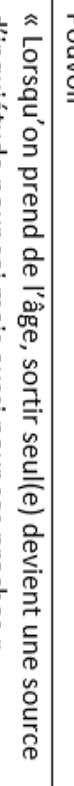 & 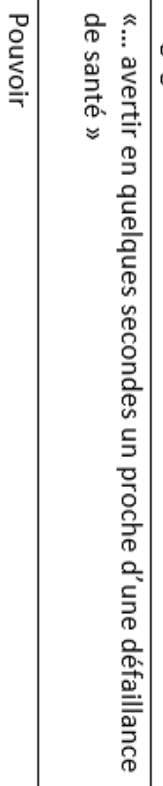 & 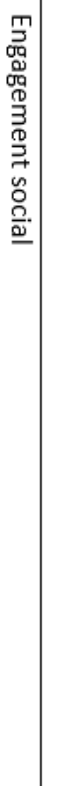 & 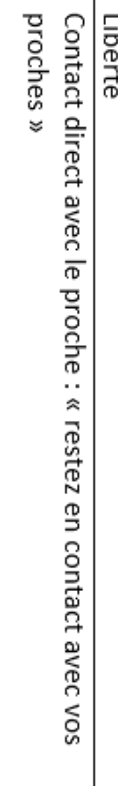 & 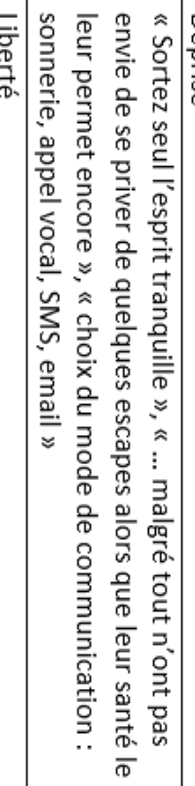 & 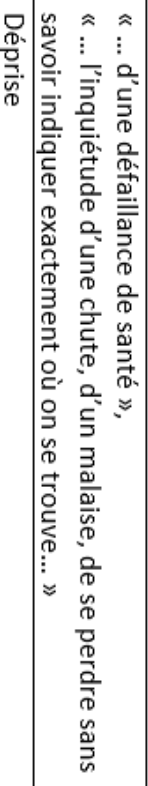 & 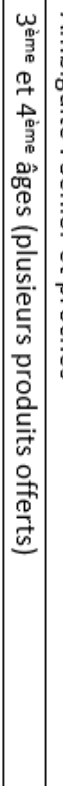 & & 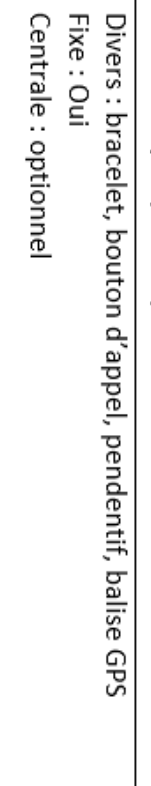 & \\
\hline
\end{tabular}




\section{TABLEAU 1}

\section{Extrait de l'analyse thématique des sites web des marques de téléalarmes}

Comme nous pouvons le voir ces antinomies reflètent la distinction entre le troisième et le quatrième âge (Hummel, 2002) que nous avons appliquée pour la sélection des séniors que nous avons rencontrés. Il nous semblait en effet intéressant de ne pas nous limiter à l'analyse du discours communicationnel mais d'interroger directement la cible qu'elle soit du troisième ou du quatrième âge afin de pouvoir prendre en compte son vécu dans nos analyses itératives.

\subsection{Les entretiens}

Le choix d'entretiens semi-structurés s'est imposé comme une évidence, car ils prennent en compte les paroles, désirs, attentes et besoins des séniors et correspondent aux nouveaux modèles d'action sociale fondés sur une plus grande réciprocité dans la relation. A la suite d'Argoux et Puijalon (2003), en reconnaissant l'existence des séniors en tant que sujets et en ne les considérant pas comme des objets, nous avons observé leurs récits comme un mouvement d'histoires individuelles au travers desquelles s'observe la vieillesse. Il nous faut préciser qu'aucun des séniors rencontrés ne souffrait de la maladie d'Alzheimer ou de démences apparentées.

Nous avons identifié les séniors en nous basant sur les variables discriminantes 7 : transferts, 8: déplacement à l'intérieur, 9: déplacement à l'extérieur et 10 : communication à distance de la grille A.G.G.I.R. (Autonomie Gérontologique, Groupes Iso-Ressources) qui évaluent le niveau de l'autonomie de la personne âgée.

Comme le mentionne le directeur du marketing et de la communication de Zembro, le bracelet-alarme vise les « jeunes séniors actifs » de moins de 69 ans qui vivent encore un état performatif au quotidien, les « séniors actifs », âgés d'entre 70 et 79 ans qui se rappellent de cet état d'activité et peuvent encore s'y projeter, et les « grands séniors actifs » de 80 ans et plus qui ont pris de la distance et considèrent la période d'activités notamment professionnelles comme révolue et le monde actuel de l'emploi différent de celui qu'ils ont connu.

Nous avons sélectionné 6 « jeunes séniors actifs », 4 « séniors actifs », 5 « grands séniors actifs », soit 15 séniors ( 5 hommes et 10 femmes) dont 2 disposaient d'un système d'alarme personnelle. Ces personnes ne connaissaient pas Zembro avant notre rencontre. Même si notre étude est qualitative et que nous ne visons pas la représentativité de notre échantillon, nous désirions avoir des personnes des deux sexes et se situant dans les trois catégories d'âge.

Nous avons élaboré notre guide d'entretien semi-directif en quatre temps : le premier dressant un portrait socio-démographique et psychographique du sénior, le 
second interrogeant son attitude par rapport à une situation fictive, soit une technique projective pour les séniors non utilisateurs d'alarme personnelle, ou par rapport au vécu expérientiel pour les 2 séniors portant un système d'alarme, le troisième accompagnant le sénior à travers sa lecture de la brochure de Zembro pour l'analyse thématique textuelle et visuelle, et le dernier questionnant le sénior sur son attitude et son comportement par rapport à l'utilisation d'un bracelet-alarme, et sur sa perception du discours communicationnel de la marque. Nous avons interprété les besoins des séniors à travers une grille objectivante et inscrit leurs récits dans un registre fonctionnel, ce qui a établi une distance entre le chercheur et le sénior, ceci pour «évincer les effets «perturbateurs » des émotions, pour techniciser les interventions et accroître leur efficacité » (Argoux et Puijalon, 2003, p.32).

Avant de présenter les résultats et analyse issus de cette collecte de données, il nous semble important de nous pencher sur la littérature autour du processus contemporain de construction sociale de la vieillesse, et sur les normes qui y sont associées.

\section{La vieillesse : une construction sociale}

Tréguer (2007) distingue trois types d'âges : l'âge biologique, le plus objectif, qui repose sur un vieillissement programmé; l'âge psychologique qui est l'âge que l'individu souhaite avoir et dépend des comportements appris et développés ; l'âge social influencé par les modèles, les représentations et les préjugés développés par la société.

Le Dictionnaire des personnes âgées, de la retraite et du vieillissement paru en 1984 distingue « les jeunes vieux », entre 60 et 75 ans, voire 80 ans, biologiquement jeunes mais socialement âgés, et les «vieux-vieux » plus âgés et pour qui «les risques pathologiques et la probabilité de vivre seuls diffèrent de façon notable », donc physiologiquement et socialement âgés (Trincaz, 2015, p.474). Nous pourrions y ajouter les «jeunes vieux », biologiquement âgés mais socialement jeunes. Cette distinction utopique masque dans un premier temps la vieillesse en jeunesse avec ses loisirs, ses activités, son autonomie et sa santé.

La vieillesse se construit sur une réalité qui comprend des éléments d'ordre biologique, démographique, politique, économique... mais elle se construit aussi sur un imaginaire culturel de représentations (Moscovici, 1984). La vieillesse est donc une construction sociale comprenant des enjeux identitaires et renvoyant inévitablement à la question de l'autonomie.

En sociologie, l'important n'est pas de définir qui est vieux ou à partir de quand on le devient, mais de décrire le processus qui désigne socialement les individus comme tels. La vieillesse, comme catégorie, est délimitée par « la distribution du pouvoir et des privilèges entre les classes et les générations » (Foucart, 2003, p.15). Elle est annoncée par l'arrêt de toute activité professionnelle et devient non seulement « un 
nouvel espace de vie pour une proportion importante de la population [mais aussi] un nouvel espace social $\gg($ Hummel, 2002, p.69).

La vieillesse n'est donc pas uniquement un état d'esprit (Henaff-Pineau, 2012), mais elle est aussi, comme le disait Simone de Beauvoir, la représentation de qui nous sommes à travers la vision que les autres ont de nous, ainsi que la comparaison aux autres. Le sénior est à même de «situer ses comportements et ses capacités, en les mettant en parallèle avec ceux des autres » (Vigouroux-Zugasti, 2014, p. 265). En comparant son dynamisme, sa motricité, ses activités, sa vivacité avec les actions que d'autres séniors, plus jeunes, du même âge ou plus âgés ont peine à effectuer, le sénior s'extrait de la catégorie des vieux (Henaff-Pineau, 2012) et se construit un nouvel équilibre identitaire.

\section{Comment bien vieillir ?}

Une première étape consiste à identifier la représentation sociale du sénior à un segment donné de son existence, et à analyser comment il interprète cette représentation qui devient pour celui qui y adhère la réalité elle-même (Humbert, 2008). Si cette succession « représentation, interprétation, adhésion, réalité » aboutit à une réalité synchrone entre celle du sénior et celle de son « cercle d'influence », un véritable dialogue peut s'instaurer, basé sur le respect de la liberté et la recherche d'un nouvel équilibre en phase avec cette nouvelle étape identitaire. Si la réalité du sénior ne coïncide pas avec celle du « cercle d'influence », une rupture peut entraîner un jeu de pouvoir et un rapport de force (Viriot-Durandal, 2012).

Comme notre société de productivité octroie peu de valeur à la vieillesse et peu de prestige aux activités des séniors, à peine visibles à l'extérieur de la famille (Arcand, 1982), certains séniors veulent rester jeunes à tout prix, au risque d'être montrés du doigt. D'autres acceptent de devenir vieux, puis de l'être, certains s'y résignent même.

Le diktat du «bien-vieillir » s'impose insidieusement dans tous les faits et gestes quotidiens du sénior et prescrit les comportements et les règles de vie conformes à son âge. Le « vieillir jeune » s'associe à des valeurs et principes puissants qui conforment les manières de vivre et de vieillir, les érigeant en modèles. L'injonction d'autonomie prend pour mot d'ordre « interdit de vieillir» (Amyot, 2012).

\section{Le sénior actif}

Le terme sénior, qui s'inscrit dans l'idéologie activiste, est issu du marketing et trouve son origine dans une approche sportive, entre le junior et le vétéran (Trincaz, 2015). On associe à ce terme des individus en pleine forme, dégagés des contraintes du travail salarié, disponibles pour leurs familles et disposant de revenus confortables qui leur permettent sorties et voyages (Tréguer, 2007). 
Nous nous référons à la définition des personnes âgées de Caradec (1999) : des personnes qui ont cessé d'avoir une activité professionnelle et dont les enfants ont quitté le nid. Les séniors s'affirment comme actifs, consommateurs, mais ils manifestent également un souci de reconnaissance et d'utilité sociale à travers des activités de bénévolat ou des aides diverses dans le cadre de la sphère familiale (Trincaz, 2015).

\subsection{Les enjeux identitaires des séniors actifs}

$\mathrm{Si}$ « vieillir, c'est se métamorphoser » (Delage et Lejeune, 2009, p.139), une double épreuve s'impose : celle de la dissociation et celle de la reconnaissance. Quand le décalage entre le ressenti et l'apparence devient trop aigu, le doute émerge. L'âge chronologique ne correspond plus à l'âge subjectif qui désigne l'ensemble des représentations cognitives associées à l'âge (Gana et al., 2004). Dans cette perspective, la jeunesse serait le temps de la coïncidence entre l'être et le paraître, la vieillesse en serait la rupture. Un sentiment d'étrangeté résulte de cette dissociation. La honte naît parfois de ces prévenances qui font ressortir la fragilité. Quant à l'indifférence, elle est cruellement ressentie. L'invisibilité s'installe. La relation aux autres devient dissymétrique et est reconnue comme telle. Certains, parmi les séniors actifs, luttent et refusent le processus physiologique comme ils refusent le retrait social. L'âge subjectif peut être considéré comme une manifestation d'un processus de défense de l'image de soi où les séniors s'efforcent de maintenir une cohérence de soi malgré les changements physiques et sociaux liés à l'âge (Moschis, 1994).

Pour Granjon (2012) qui se réfère à Honneth (2000), la société est constituée de trois sphères normatives relatives à la reconnaissance mutuelle, au respect en tant que sujet juridique et au partage des pratiques : l'amour, le droit et l'estime de soi. La reconnaissance de soi par les autres est cruciale pour l'estime sociale de soi et dans la construction identitaire. Dans le cas contraire, le sénior subit le déni du corps social dont il ne fait plus partie, rejet social dont souffrent les retraités (Vigouroux-Zugasti, 2014) qui se retrouvent à un moment où le cercle social se réduit (éloignement de la famille, mort des proches), réduction des relations sociales qui encourage l'isolement (Caradec, 2012). Vieillir implique un réaménagement de ses activités et de ses engagements (Caradec, 2004).

\subsection{La déprise}

Enjeu fondamental, la déprise est « liée à une perte de capabilité ou de « pouvoir de » poursuivre certains engagements antérieurs » (Lavoie et al., 2011, p.64).

Selon Sen (1999), la capabilité désigne un ensemble de vecteurs de fonctionnements, qui reflètent la liberté effective dont dispose actuellement la 
personne pour un type de vie donné. Alors que la capacité peut s'actualiser ou non, la capabilité est réelle et actuelle.

Pour Amyot, la déprise s'observe par l'« inversion des rapports d'autorité [...] dont la manifestation la plus tangible sera la prise de pouvoir de l'entourage sur les conditions de vie de la personne » (2012, p.132). A terme cette inversion aboutit au processus de non-reconnaissance et de privation des droits et des ressources qu'est l'exclusion, et qui se réalise «à l'encontre de certains segments de la population, [...] à travers des rapports de forces entre groupes aux visions et aux intérêts divergents » (Billette et Lavoie, 2010, p.5).

La vieillesse est souvent associée à la déprise, le fait d'abandonner certaines activités pour se concentrer sur les plus significatives compte tenu de l'identité que le sénior tente de préserver (Lavoie et al., 2011). Pour que le sénior puisse accepter sa nouvelle situation identitaire, il faut qu'il ait intériorisé de nouvelles normes : la prise de conscience d'autres limites, l'acceptation de ne plus pouvoir faire certaines choses. Or, ce « jamais plus » renforce l'angoisse du déclin (Trincaz, 2015).

\subsection{L'autonomie}

Alors que la dépendance affiche les notions de déficience, d'incapacité, de soutien, de relation d'aide, d'échange..., l'autonomie fait l'apologie de la potentialité, la capacité, l'indépendance, le contrôle, le libre-arbitre, elle crée une vision positive de la vieillesse. Il n'y a pas encore de « transfert du pouvoir de décision » (Amyot, 2012, p.218).

Ce transfert engage le verdict de la perte d'autonomie : ne plus pouvoir agir par soimême. A titre individuel, il s'agit d'un constat de perte ; pour le grand âge collectif, il s'agit d'une précaution à des fins de protection ouvrant le débat sur le droit au choix et le droit au risque au grand âge. Le contrôle social va s'exercer ici à partir de la représentation de la vieillesse. Quand le contrôle prend le dessus, le corps social réduit l'univers existentiel du grand sénior aux actes élémentaires de la vie quotidienne utilisés pour calculer le niveau de dépendance. (Amyot, 2012).

Comme les craintes liées aux responsabilités nourrissent les pratiques sécuritaires, et comme la notion de responsabilité est générale, morale, philosophique, avant d'être juridique, les professionnels ou les proches des personnes âgées sont sur la défensive. Il y a une tendance générale à notre époque à confondre autonomie et responsabilité. Cette dernière est calculée assez intuitivement par les tiers qui évaluent les conditions définissant le risque par rapport aux éventuelles sanctions sociales : la mise en sécurité du sénior pour se sécuriser soi-même, le dilemme entre la non-assistance à personne en danger et l'intervention abusive qui prive le sénior de sa capacité de choix. L'enjeu est la prise de conscience de la complexité des situations vécues par les séniors et leurs proches (Amyot, 2012). Mais comment vieillir dans une société qui présente son rapport au monde excluant la vieillesse? 
Les séniors disposent de temps qui permet la réalisation de projets jusqu'alors repoussés faute de temps. Les discours communicationnels de biens et de services visant les séniors pourraient être positivés. De plus, les prestataires de biens et services doivent composer avec une dualité dans la cible. D'une part les séniors en phase avec leur identité qui remettent leur vie en perspective, font une relecture de leur vie par l'usage d'une parole réflexive, et dont l'enjeu est la construction d'un savoir sur la vieillesse en intériorité. D'autre part les séniors qui se mettent en scène par l'usage de la parole représentée, font un travail d'élaboration pour passer du niveau individuel au niveau collectif, soit une extériorité qui permet l'objectivisation mais dresse une distance entre le sénior et la vieillesse qui n'est pas sa vieillesse (Argoux et Puijalon, 2003).

\section{Entre éthique et stratégie : quand Zembro communique vers les séniors}

Quelle est la position du communicant face à cette cible particulière ? Doit-il jouer le jeu de la société et légitimer une vision de complicité idéale souhaitée par les proches permettant de surveiller le sénior à distance ? Peut-il ou doit-il contribuer à modifier la place du sénior dans notre société ? Comment s'adresser à la fois aux séniors et aux proches quand on sait que ceux-ci seront bien souvent les prescripteurs du service et qu'ils participent à son autonomie si l'on conçoit celle-ci comme étant solidaire ou relationnelle (Ennuyer, 2013) ? Quel choix d'éthique fait Zembro entre celle - utilitaire - basée sur les conséquences des actions et celle - kantienne - basée sur le devoir des professionnels par rapport à la société (Fawkes, 2015) ? Quelle est la responsabilité du communicant dans la construction de ces représentations sociales qui font du sénior un «individu collectif» (Thomas, 2005) ? Le port d'un braceletalarme peut-il permettre au sénior d'accepter sa nouvelle étape identitaire ?

Comme la majorité des entreprises ou des associations qui vendent ou louent des systèmes d'alarme, Zembro se positionne vis-à-vis de la définition de l'identité des aînés, dans laquelle le chez-soi devient pierre angulaire de la vie sociale (Clément et al., 2004). Développée dans une perspective de complémentarité mais non de substitut du contact humain, ce type de dispositifs est au service du mieux-vivre, du maintien à domicile, permettant d'agencer un environnement favorisant l'autonomie (Cornet et Carré, 2008). Zembro commercialise un système d'alarme mobile, porté par le sénior, intégrant un système de transmission à distance qui permet de déclencher des alarmes volontaires. L'appel est successivement transmis vers les smartphones des connexions 2 introduites dans le «système ». En ne s'inscrivant pas dans le

2 Les connexions sont le plus souvent des membres de la famille, mais peuvent être également des amis, voire des voisins. 
«jeunisme » des TIC, Zembro se distingue de ses concurrents. Aucun prérequis n'est imposé au sénior pour son utilisation et la technologie s'adresse tant à lui qu'à ses proches. Dès qu'une des connexions réagit, le sénior peut l'informer de la situation. Ce système n'est pas intrusif car, comme le rappelle Bert Duhamel, directeur marketing et communication de Zembro, il ne sera possible de localiser le sénior qu'à sa demande : on ne peut le surveiller sans son accord. La géolocalisation lui permet de se déplacer à l'extérieur. Le système a été pensé pour ne pas être identifié comme tel pour que le sénior ne se sente pas déprécié (une montre, un bijou, mais pas une alarme).

Comme l'indiquent Cornet et Carré (2008), l'éthique est intégrée dans l'approche qualité de ce système. Bert Duhamel illustre cela quand il évoque la conception conviviale et simple des interfaces, les notices d'emploi, le respect des données personnelles, de la dignité et de la liberté des usagers. Les normes concernant la manière de concevoir la place de la personne âgée en perte d'autonomie sont donc intégrées au système. Ce qu'il va permettre ou pas de réaliser s'inscrit dans une approche qui dépasse la simple question technique. Comme le précise le directeur marketing : «Le bracelet Zembro permet au sénior actif d'avoir le sentiment de vivre comme avant, de faire comme si rien n'avait changé dans sa matérialité physique. »

Le bracelet Zembro mise sur le vieillissement sur place (aging in place). Il contient la promesse du «bien-vieillir », préservation de l'état au cours d'un processus qu'est le vieillissement (Trincaz, 2015), mais est polarisant car il s'adresse au sénior dans son état d'aujourd'hui ou de demain comme le dicte la société, donc il redéfinit son identité de «jeune-vieux » vers la position de «vieux ». Le bracelet soulève une tension entre sécurité et liberté.

Le dispositif de télé-alarme Zembro vise à maintenir, voire même (re)créer du lien social permettant aux séniors d'échapper au phénomène de déprise et $d$ 'isolement. Paradoxe identifié par Caradec et Eve (2002) dans l'attention particulière à l'égard de la vie privée des autres (le souci de ne pas déranger) et de soi (son indépendance) et la possibilité d'être joignable. Comme cela s'est vérifié dans nos entretiens, c'est souvent l'utilisation " pour les autres » qui confère une utilité à un objet technique et rend sa dépense justifiable.

Zembro avait le choix entre deux logiques : celle de «prise en charge » ou celle d' «accompagnement». La logique de «prise en charge » suppose la prédominance de la parole de la marque et de celle de la connexion sur celle du sénior. C'est une logique explicative, applicable à tous les individus, dans une verticalité communicationnelle. La logique d' "accompagnement» permet une rencontre entre des points de vue d'égale valeur. C'est une logique compréhensive, portée sur le cas par cas (Argoux et Puijalon, 2003, p.33), dans une approche horizontale de la communication visant la réduction de l'isolement par la construction de lien social, le partage d'expérience par le développement de communauté(s), l'illustration de la facilité d'usage et le respect des choix individuels (Duvernay, 2011). 
C'est la logique «d'accompagnement» qui prévaut: selon le directeur du marketing et de la communication de Zembro, le double discours (vers le sénior et vers ses proches) a pour avantage d'interpeller le proche qui est ou sera lui-même un sénior actif. Ainsi, la stratégie de communication sert les deux, l'un à court terme, l'autre à moyen terme. Dans la brochure de présentation, on s'adresse tantôt au sénior comme à un adulte responsable : «Zembro me donne toute la liberté », tantôt au sénior via ses proches, comme si les décisions prises à son égard ne le concernaient plus que de façon indirecte, nécessitant une médiation: «Vous êtes certainement nombreux à connaître cette situation : vos parents, votre sœur, votre frère ou vos amis vieillissent et vous vous inquiétez ». La communication de Zembro joue sur les deux tableaux.

Le fait d'également s'adresser directement au sénior et pas uniquement à ses proches est un élément déterminant dans le rôle du communicant dans la représentation sociale véhiculée par la société. Au-delà de l'aspect stratégique, si l'on conçoit la communication comme organisante, ou comme une performance de la culture, s'adresser au sénior comme un sujet et non un objet de soins contribue à façonner son image et sa place au sein de la société. Zembro a choisi de mettre en avant dans sa communication visuelle et dans son discours le visage positif de la vieillesse permettant au sénior de garder sa fierté sans être stigmatisé, de la vivre de manière active, autonome et libre en faisant partie de la société. Pour lever les freins psychologiques de sa cible, le directeur marketing et communication de Zembro respecte comme principe et règle éthiques de conception et de mise en service de ne pas souligner la face négative de la vieillesse qui écarte le sénior de la société sous le couvert de la passivité, de la dépendance, du contrôle, voire de la culpabilisation de vieillir. Le bracelet est présenté comme un assistant permettant de continuer à être actif et présent au sein de l'espace public, comme un outil offrant de conserver son autonomie malgré un corps pouvant présenter des défaillances : «(...) il garantit liberté, indépendance et tranquillité d'esprit si chères à tous ». D'autres dispositifs étudiés, Télésecours par exemple, vont de leur côté mettre davantage en avant le côté négatif de l'avancée en âge en évoquant directement les situations difficiles : "Simple et efficace : nous vous proposons une aide immédiate pour vous secourir à domicile en cas de chute, de malaise... ».

Si l'approche fait partie d'une stratégie marketing visant à rejoindre directement sa cible et à lui vendre son produit, il n'en demeure pas moins que cette stratégie a un impact sur l'image du sénior tant pour ce dernier (âges biologique et psychologique) qu'au sein de la société (âge social). 


\section{Les séniors ont leur parole : résultats des entretiens}

\subsection{La représentation identitaire}

Quel est l'impact du port d'un bracelet-alarme sur la représentation identitaire ? Si, par un comportement et une réactivité supérieurs à ceux d'autres séniors - plus jeunes, du même âge ou plus âgés (Henaff-Pineau, 2012) - le sénior parvient à s'extraire de la catégorie des vieux (l'âge psychologique), le port du bracelet l'y (re)plonge (l'âge biologique) : «Avec ça au bras je me sentirai vieille » (F66). Le jugement et le regard des autres prennent toute leur importance: «Je serais taxé de vieux» (H75); "Quelqu'un qui a ça alors on pense "celui-là il a besoin d'aide" » (F59). Les séniors interrogés se représentent à travers la comparaison aux autres et la vision qu'ils pensent que les autres ont - ou auront - d'eux (l'âge social).

Porter un bracelet-alarme, c'est devoir consentir aux restrictions imposées par son corps (F72), c'est afficher un corps qui ne répond plus aux attentes. « Non, je sais à peine marcher, enfin je sais marcher mais difficilement, je tombe souvent, oui, ça me sert (elle montre son déambulateur), ça me sert à me bouger... » (F93).

Certains séniors vivent cette violente métamorphose : «Je ne me reconnais plus, je n'arrive pas à réaliser que j'ai 83 ans » (F83). Ils sont confrontés à la recherche d'euxmêmes parmi les trois images : celle que l'on se fait de soi, celle que le miroir renvoie et celle que les autres s'en font.

La peur ou la honte s'ajoutent à l'épreuve de la dissociation et de la reconnaissance qui mène alors la personne à vivre en marge afin de ne pas subir l'opprobre des autres. «J'aimais lire, mais quand je tourne la page, je sais plus ce que j'ai lu, je dois recommencer. J'ai abandonné la bibliothèque tournante » (F83). Le corps devient parfois un alter ego exigeant et tyrannique : «Je dois prendre des médicaments trois fois par jour » (F88). Sa présence de plus en plus envahissante compense le décès des pairs d'âge et remplit le vide social qui s'installe : «J'ai d'autres amis bien sûr mais plus trop, tous mes amis s'en vont doucement rejoindre le ciel mais moi, on m'oublie (rires) » (F93).

\footnotetext{
Avant on allait tous les dimanches voir les grands-parents, on allait chez la maman de mon mari et on repassait par chez ma sœur, on restait souper, mais maintenant on y va seulement pour les grandes occasions, puis voilà. (F71).
}

Dans le discours des séniors actifs rencontrés, on a de nombreuses références au temps passé comme à un temps révolu, des choses que l'on faisait, que l'on aimait mais que l'on ne peut plus faire, que l'on ne fera plus jamais. La notion d'acceptation d'une nouvelle situation identitaire prend ici tout son sens. Les recherches sur la téléalarme ont d'ailleurs montré que son adoption ou son refus par les personnes âgées 
ne pouvait se comprendre que dans le cadre de la négociation de leur propre vieillissement. La téléalarme est signe de la dépendance et les stratégies d'évitement de la stigmatisation ont été souvent relevées, la principale étant de ne pas porter l'alarme sur soi (Jani-Lebris et Luquet, 1997). Nous avons pu le vérifier lors de nos entretiens. Acceptation et adoption sont liées à la mobilité, à la condition physique et psychique, à la situation personnelle : «Je l'envisagerais si mon épouse devait partir avant moi » (H86), à l'intériorisation de la nouvelle étape identitaire.

\subsection{Le diktat du « bien-vieillir»}

Un individu est dans la vie ce qu'il fait dans la vie. Dans notre société, orientée vers la productivité, le statut social dépend du salaire. Le travail productif servant de référent, « exiger des personnes âgées qu'elles se consacrent aux loisirs, c'est presque leur demander d'aller vivre sur la lune » (Arcand, 1982, p.22).

Nous constatons au fil des entretiens que les séniors n'expriment plus ce mal-être soulevé par Arcand. Leurs activités sont multiples et elles sont le prolongement de celles qu'ils souhaitaient avoir lorsqu'ils étaient dans la vie active mais n'avaient pas eu le temps de vivre pleinement : concerts, visites de musées, théâtre, cinéma, chorale, promenades, matchs de foot, cours, jardinage, entretien de la maison, cuisine, voyages, visites aux amis, à la famille, garde des petits-enfants, volontariat, engagement social, pastoral et même «visite quotidienne au bureau pour s'occuper des mauvais payeurs et faire le travail qui traîne » (H75).

Nous retrouvons donc la distinction de Trincaz (2015) entre les « jeunes-vieux » et les « vieux-vieux », ou celle de Hummel (2002) entre le troisième et le quatrième âge qui toutes deux maquillent dans un premier temps la vieillesse en seconde jeunesse, avec son cortège de loisirs, de santé et d'autonomie.

Insidieux le diktat du «bien-vieillir»? Les séniors actifs ne le vivent pas consciemment comme tel, mais ils estiment qu'il est de leur devoir d'être actif, de ne pas se laisser aller et de profiter pleinement du temps libre tant que la santé le leur permet car «c'est si vite arrivé » (H65). Le bracelet peut les accompagner dans ces réalisations. «Moi ça ne me dérangerait pas de le porter pendant mes promenades... Je suis déjà tombée sans savoir pourquoi ni comment... Je pourrais avertir quelqu'un. Je ne devrais plus me demander si ça va aller. » (F60).

\subsection{La déprise}

Dans le cas du sénior actif, il est plutôt question d'une semi-prise de pouvoir car on ne le dépouille pas «de son propre projet de vie » (Amyot, 2012, p.132), il ne doit pas (encore) refouler ses désirs ni accepter les desseins et les exigences des proches ou des professionnels : le coup de force est retardé. Notons toutefois qu'avec un dispositif de téléalarme la personne garde un certain pouvoir de décision : l'acquérir 
ou pas, le porter ou non, décider ou non d'appuyer en cas d'urgence. «Je n'ai pas appelé tout de suite... juste le lendemain matin, pour ne pas déranger... » (F86). La déprise peut être volontaire, due au regard de la société (Vigouroux-Zugasti, 2014) : «A mon âge on ne va plus comme ça au cinéma; c'est plein de jeunes» (F91), ou imposée par le physique comme l'illustre la réaction par rapport à la photo de la brochure de Zembro montrant un sexagénaire juché sur un escabeau et qui change une ampoule (Figure 1 : Brochure Zembro).

Oui, ça, c'est pas malin. Quand tu es dans un état où tu sens que ça ne va pas très bien, tu ne vas pas monter sur une échelle. A un moment donné il faut savoir arrêter donc tu ne fais plus des choses comme ça hein... (H78).

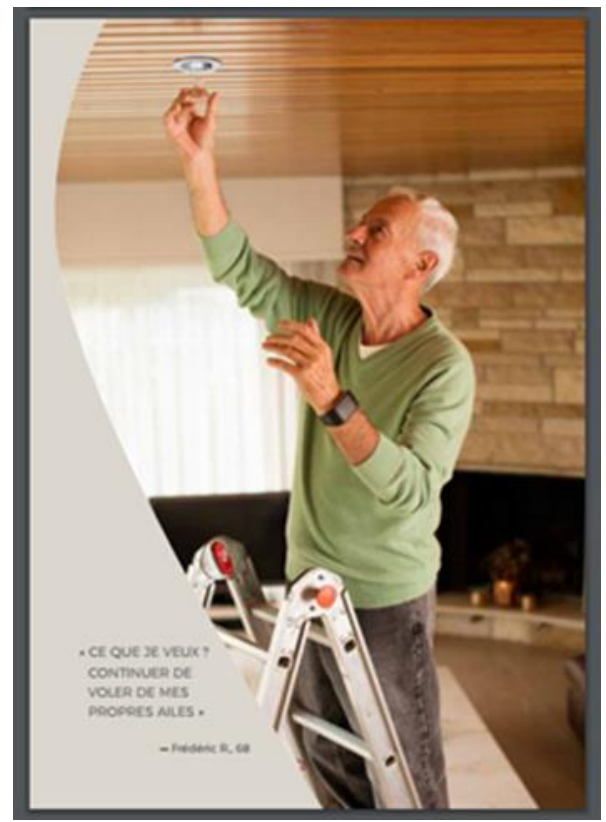

\section{FIGURE 1}

\section{Page 9 de la brochure de présentation de Zembro 2015}

Les séniors actifs, non utilisateurs de tels dispositifs, ont tendance à ne pas se considérer comme déjà vieux. Cette situation de déprise ne pouvant s'envisager que dans le futur plus ou moins proche, mais pas immédiat, il leur est plus aisé de parler du système lié au cas fictif (la parole représentée) que par rapport à leur propre vécu (la parole réflexive). «C'est délicat de parler de ça [le bracelet-alarme] : c'est dire à l'autre qu'il n'est plus capable de se gérer tout seul » (H78). 
La déprise renvoie à la perte de pouvoir orienter sa vie et son environnement comme on l'entend (Lavoie et al., 2011) : «Tu vas beaucoup te promener ? (soupir) Pas tellement. Avant oui, mais comme mon mari a dif... n'aime plus marcher, on n'y va plus tellement et moi toute seule, je n'aime pas trop. J'y vais parfois mais... » (F71).

Nous retrouvons cette lecture dans les entretiens avec les séniors portant un tel dispositif :

Oui, quand je m'éloigne je le prends. C'est un collier. C'est très désagréable ce collier parce que il est (incompréhensible) alors je le pose là mais si je dois aller à la salle de bains, n'importe quoi, quand je me déplace, je le prends. (F93)

Oui, l'appareil, je le mettais pas quand il y avait quelqu'un non hein, mais quand il n'y avait personne je le mettais le soir près de ma table de nuit et alors quand je me levais je le mettais direct autour de mon cou, ça j'ai toujours fait puisque je l'avais quand j'ai tombé. (...) J'aimais bien en collier et je le mettais en dedans. Oui comme ça, ça se voyait moins. (F86)

La déprise mène à l'exclusion :

J'aimais bien rouler en voiture, mais à 83 ans on m'a dit que c'était trop risqué ; ça m'a fait tout drôle. Maintenant encore je regrette. Mais bon... Je ne peux plus voir qui je veux quand moi j'ai envie... (F83).

L'alarme personnelle confronte le sénior à son présent et à son futur proche : le regard sur soi et la prise de conscience d'une insécurité croissante, et le regard des autres avec l'appréhension de devenir un poids pour les autres, de perturber leur vie privée : «Faire venir les gens inutilement si ça se déclenche par hasard, et ça arrive et c'est très ennuyeux pour la vieille personne que son enfant se déplace pour rien » (H79).

\subsection{L'autonomie}

Le jour de la retraite, le sénior peut se retrouver pour la toute première fois sans ordres. Ce changement de condition brutal peut être déroutant et certains retraités s'imposent toute une série de contraintes plutôt arbitraires pour à nouveau s'astreindre à un quotidien ordonné et régularisé (Arcand, 1982) : création d'une auto-servitude volontaire comme la prônait la Boétie.

Quel rôle peut jouer une alarme personnelle dans cette nouvelle situation, quelles attentes peut-elle combler? «J'avais moins peur, j’étais plus rassurée ... ça oui, je ne l'oubliais jamais et quand j'allais jusque chez la voisine je le mettais aussi » (F86) ; «Un peu rassurant disons quoi que tant qu'il ne vous arrive rien on ne pense pas au mal, on ne pense pas qu'il va arriver des choses » (F93). 
Sécurité, ou sentiment de sécurité pour soi ou pour ses proches, tranquillité, maintien de l'autonomie, possibilité de vivre plus longtemps chez soi et aide directe et rapide d'une personne de confiance en cas d'urgence sont les avantages perçus par les non-utilisateurs. Ils illustrent la vision positive de la vieillesse, lors le transfert du pouvoir de décision n'a pas encore eu lieu.

\subsection{Accepter une nouvelle situation identitaire}

Les entretiens confrontent le sénior à sa situation identitaire avec un maximum d'objectivité, amenant la réalité de son quotidien au cœur des échanges et soulevant des faits pas nécessairement partagés jusqu'alors. En sélectionnant ses confessions, le sénior fait le tri entre « le dicible et l'indicible » et mène une réflexion sur sa propre construction de la vieillesse.

Les séniors qui vivent consciemment leur situation identitaire et l'acceptent, voient ou s'imaginent les avantages plus que les inconvénients qu'offre l'alarme personnelle et les expriment par le biais de la parole réflexive. Ils intériorisent l'éventualité d'un usage par le biais d'une mise en perspective de leur propre vie. Quand nous les interrogeons sur la communication de Zembro, suite à la découverte de la brochure, ils l'interprètent comme éthique car présentant le réel avec respect.

Je connais des patientes qui portent une alarme un peu pareille, qui appelle un poste si on ne se sent pas bien. Comme ça elles ne doivent pas rester à attendre que quelqu'un vienne les voir. Elles peuvent aller en ville, boire une tasse de café, aller chez le boulanger... Enfin faire comme avant sans avoir à demander de l'aide à tout bout de champ. C'est vraiment important pour elles (F61).

L'utilisation de la parole représentée fait appel à une mise en scène, à une extériorisation de l'usage d'un système d'alarme, et indique une substitution consciente ou non - du vécu par une projection de vécus mis en scène « hors du sénior ». La vieillesse n'étant pas considérée comme une étape qui l'amoindrit, tout instrument y référant devient synonyme de perte d'autonomie et la perte d'indépendance est identifiée comme incompatible avec sa vie.

Les enfants n'appellent pas souvent : ils pensent sans doute « on ne l'entend pas, donc ça va ». Mais je vois mes frères et sœurs au moins une fois par mois. On parle de plein de choses. Mais ça jamais on n'a parlé d'un système d'alarme ! On n'en a pas besoin. Pourquoi en parler? On fait encore toujours la même chose qu'il y a 5 ans. On est de bonne souche (rires) (F90).

Nous sommes au cœur du débat portant sur le droit au choix et le droit au risque des séniors. Le contrôle social s'exerce à partir de la représentation qu'a le tiers de la vieillesse. Les avis des séniors interrogés sur ce tiers influenceur sont partagés : enfant, partenaire, proche, médecin ou même voisin. Mais tous sont d'accord pour que 
ce soit quelqu'un de confiance avec qui les rapports sont vrais et étroits, quelqu'un qui ne viserait pas à réduire l'espace vital du sénior sans raison. Les situations présentées dans la brochure sont en adéquation avec leur ressenti. Les séniors sousentendent la volonté d'avoir un droit de réponse. Tous revendiquent le droit au choix et les non-utilisateurs le droit au risque.

Mais peut-on circonscrire au risque à tout prix ? La stratégie de communication peut-elle, comme un miroir aux alouettes (Guillemard, 1978), renforcer le « droit de choisir et de prendre des risques [...] dénié à ceux qui incarnent la vieillesse » (Amyot, 2012, p.131) ? Zembro mise sur cette approche de la liberté dans son e-mailing promotionnel pour la fête des mères (29 avril 2016). Un des témoignages y promeut la remise en circulation routière sans sembler se soucier de la sécurité des autres. Les choix de communication de Zembro quand elle s'adresse directement au sénior associent la liberté au bracelet. Le porter permet de voyager, de continuer à bricoler, de partir en promenade... tout en rassurant l'entourage. Cette posture de facilitateur d'une période de vie à une autre nous mène à questionner la position du communicant face à cette cible particulière. Doit-il jouer le jeu de la société et légitimer une vision de complicité idéale souhaitée par les proches permettant de surveiller le sénior à distance ? Peut-il ou doit-il contribuer à modifier la place du sénior dans notre société ? Comment s'adresser à la fois aux séniors et aux proches quand on sait que ceux-ci seront bien souvent les prescripteurs du service et qu'ils participent à son autonomie si l'on conçoit celle-ci comme étant solidaire ou relationnelle (Ennuyer, 2013) ? Quel choix d'éthique fait Zembro entre celle - utilitaire - basée sur les conséquences des actions et celle -kantienne - basée sur le devoir des professionnels par rapport à la société (Fawkes, 2015) ? Quelle est la responsabilité du communicant dans la construction de ces représentations sociales qui font du sénior un «individu collectif » (Thomas, 2005) ? Le port d'un bracelet-alarme peut-il permettre au sénior d'accepter sa nouvelle étape identitaire?

Nos entretiens nous renvoient une vision très nuancée. Par son approche et son choix sémantique, Zembro respecte l'autonomie et l'intégrité du sénior. L'usage que ce dernier peut faire du bracelet-alarme - sous la douche, en voyage - lui permet de maintenir son état actuel d'indépendance tout en augmentant le sentiment de sécurité, tant pour lui que pour ses proches. Illustrer l'expérientiel des deux parties - sénior et enfants ou petits-enfants - est perçu comme positif et bienfondé :

Peut-être que si mes enfants devaient m'en parler que j'écouterais ce qu'ils ont à dire. Pas que je le ferais [acheter le bracelet-alarme : c'est moi qui dois continuer à décider pour moi. C'est pas à mes enfants de dire ce qui va se passer avec moi » (F90). «Il n'y a pas de mal à se faire aider, mais il faut protéger la vie privée ! Cette géo-localisation ne peut pas être accessible à n'importe qui (H65).

Mais comme le confiait Bert Duhamel (directeur marketing et communication, Zembro), un utilisateur ôtait son bracelet chaque fois qu'il rendait visite à son amie 
pour que ses enfants ne le sachent pas. Le concept antinomique de liberté-contrôlée joue un rôle clé dans l'acceptation ou le rejet d'un système d'alarme : les séniors actifs veulent rester aux manettes.

Si les témoignages et les situations décrites dans la brochure sont réalistes (Figure 2 : Brochure Zembro), il est impossible de décrire tous les cas de figures. Sans l'idéaliser, Zembro ne montre le visage actif de la vieillesse que dans des situations de confort matériel. De plus, Zembro ne réfère qu'aux séniors comme utilisateurs alors que ce service serait opportun pour qui a un problème de santé mais souhaite rester à domicile : «Ils devraient faire de cette alarme un produit normal, comme ces sièges-ascenseurs : personne ne trouve ça déplacé » (F60).

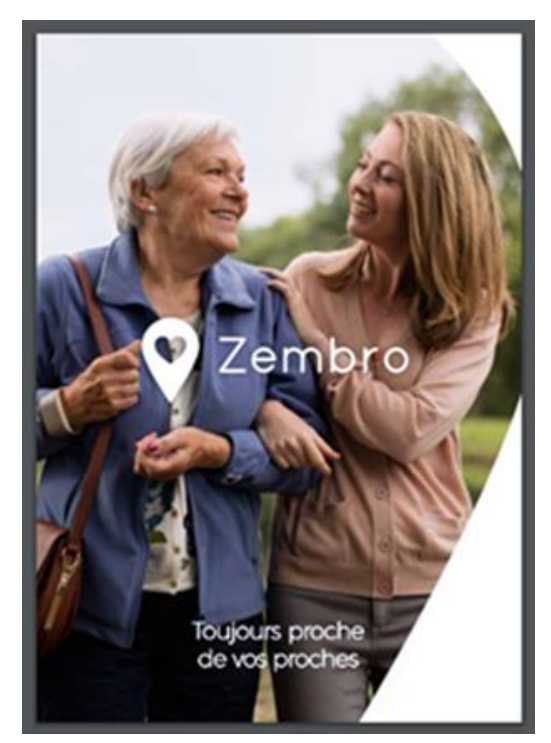

\section{FIGURE 2}

\section{Page 1 de la brochure de présentation Zembro 2015}

La communication de Zembro se situe à un niveau d'éthique utilitaire où ce sont les conséquences de l'action, pour la personne âgée et pour ses proches, qui déterminent sa valeur morale. Le produit et sa communication sont éthiques puisqu'ils vont permettre d'éviter les risques tout en maintenant un maximum de liberté pour le sénior. Mais comment passer d'un choix d'éthique utilitaire, donc basée sur les conséquences, à un choix d'éthique plus kantienne basée sur un projet de société plus global ? Il semble que « le nouveau paradigme d'une vieillesse active et réussie est 
mieux adaptée au consumérisme qu'aux solutions que devraient apporter notre société aux questions sociales et environnementales » (Hopflinger, 1995, p.96).

\section{Nos conclusions}

Par son discours, une organisation comme Zembro participe à la construction sociale de la « vieillesse » ainsi qu'aux représentations collectives entourant la notion de « sénior actif ». Zembro ne tient pas le discours de l'idéologie oppressive, idéologie dominante qui valorise la jeunesse et qui déclare que les vieux sont moins virils et les vieilles moins belles dans une société où la plupart des vieillards sont malades, pauvres, immobiles, inutiles, seuls et tristes (Guillemard, 1978). Zembro ne réfère pas à une invention simpliste et inadéquate de communicateur de masse en quête d'un stéréotype facile de la vieillesse, à une utilisation de l'âgisme implicite qui dépeint le sénior en termes nuancés et ambigus minimisant son pouvoir d'agir en le décrivant comme un individu chaleureux mais vulnérable et fragile (Lagacé et al., 2011). Il pourrait pourtant le faire sans nuire à sa stratégie de communication si, comme d'autres marques, il prenait pour cible les proches ou les professionnels de santé.

Les entretiens avec les séniors confirment l'approche respectueuse mais soulignent le souhait d'un discours plus direct utilisant moins les intermédiaires : le sénior actif doit pouvoir définir et décider des actes et des activités qui construisent sa vie. Intégrer les proches dans la communication est un élément positif, mais l'autonomie du sénior doit être prioritaire.

Par le choix d'une communication s'adressant tant au sénior qu'à ses proches, Zembro se positionne dans une conception de l'autonomie vue comme relationnelle. La personne est capable de prendre des décisions sur ce qui est bon pour elle, mais cela ne signifie pas pour autant qu'elle soit totalement indépendante et qu'elle n'ait pas besoin de ses proches pour prolonger cette autonomie, pour réaliser ce qui compte réellement pour elle. Dans les entretiens, ressort le fait de se retrouver seul - ce qui marque une rupture, un événement biographique important, comme une transition de vie, transition identitaire dans laquelle l'objet est un support chez G. Mac Cracken (1987) - et le fait d'avoir fait une chute ou de craindre d'en faire une.

Les séniors actifs non utilisateurs ont tendance à associer l'acquisition d'une alarme personnelle à une situation de déprise, et celle-ci ne peut que s'envisager dans un futur plus lointain que proche. Pour les séniors conscients de la déprise inévitable, Zembro pourrait figurer parmi les marques de leur « evoqued set», portfolio de marques par catégorie de produits ou services. Leurs proches peuvent légitimer le bracelet en l'évoquant. Le caractère rassurant du dispositif tant pour soi que pour ses proches est un argument porteur du maintien de l'autonomie comme antidote à la dépendance, de la construction d'un savoir sur la vieillesse en intériorité.

Ceux pour qui la vieillesse se traduit par la parole représentée, qui l'extériorisent et l'objectivent pour qu'elle ne soit pas leur vieillesse, ces séniors-là affichent une 
attitude plus défensive. Toutefois, comme tous vivent chez eux de manière autonome, la réalité à laquelle ils adhèrent et qui est l'interprétation de leur représentation, ne s'oppose pas (encore) à celle de leurs proches. Aussi, même si les réalités divergent, une rupture entraînant un jeu de pouvoir et un rapport de force n'a pas (encore) lieu d'être. Pour ces séniors en butte avec leur propre identité et accroché à leur âge subjectif, Zembro est perçu comme un intrus qui perturbe le cours tranquille du quotidien. Tant qu'aucune situation «à risque » ne s'est présentée, le sénior est en droit d'estimer que le système est superflu. L'utilité n'apparaît souvent qu'après-coup, lorsque les séniors ont ajusté leurs pratiques pour incorporer l'innovation (Caradec et Eve, 2002).

Zembro projette une image du sénior qui lui permet d'envisager un avenir pas uniquement centré autour de la dépendance et de la vulnérabilité, mais qui lui offre de rester en prise avec les évolutions de la société en «vieillissant jeune ».

Nous rejoignons Argoux et Puijalon (2003) pour qui réduire le sénior à n'être qu'un objet de soins, c'est jeter un discrédit sur l'expérience existentielle du vieillissement alors que l'écouter c'est redonner une légitimité à cette expérience. En intégrant le jeu de la société qui légitime une vision de complicité idéale souhaitée par les proches mais sans surveillance à distance automatique, le dispositif de téléalarme permet au sénior de garder le contrôle en avertissant la personne de son choix. En s'adressant au sénior et prenant en compte ce qu'il a été, mais aussi et surtout ce qu'il est, le communicant peut contribuer à modifier la place du sénior dans notre société. En s'adressant directement à lui, en proposant une communication horizontale (Duvernay, 2011) mettant en scène et reprenant le témoignage d'autres séniors auxquels il pourra s'identifier, la marque va permettre de réduire l'isolement en créant du lien social, le sentiment de s'inscrire dans une communauté. Le sénior, convaincu par la facilité du dispositif via des illustrations d'usage aura également davantage le sentiment que ses choix individuels seront respectés. 


\section{Bibliographie}

Amyot, J.-J. (2012). Vieillesse, contrôle social et idéologie sécuritaire. Entre autonomie et dépendance. Vie sociale, 1, 125-143.

Amyot, J.-J. (2014). Innommable et innombrable: De la vieillesse, considérée comme une épidémie. Paris : Dunod.

Arcand, B. (1982). La construction culturelle de la vieillesse. Anthropologie et Sociétés, 6(3), 7-23.

Argoux, D. et Puijalon, B. (2003). Enjeux et limites d'une prise en compte de la parole des vieux. Gérontologie et société, 3(106), 23-39.

Balard, F. (2013). «Bien vieillir» et «faire bonne vieillesse ». Perspective anthropologique et paroles de centenaires. Recherches sociologiques et anthropologiques, 44(1), 75-95.

Bawin Legros, B. et Casman, M.-T. (2001). Vieillir au féminin : quiétude ou inquiétude ? Cahiers du genre, 2(31), 149-165.

Billette, V. et Lavoie, J.-P. (2010). Introduction. Vieillissements, exclusions sociales et solidarités. Dans M. Carpentier, N. Guberman, V. Billette, J-P. Lavoie, A. Grenier et I. Olazabal (dir.), Vieillir au pluriel. Perspectives sociales (p.1-22). Québec : Presses de l'Université du Québec.

Caradec, V. (1999). Vieillissement et usage des technologies. Une perspective identitaire et relationnelle. Réseaux, 7(96), 45-95.

Caradec, V. (2004). (Dé)prise, (dés)intérêt et étrangeté au monde. Vieillir après la retraite. Approche sociologique du vieillissement. Paris : PUF.

Caradec V. (2012). Sociologie de la vieillesse et du vieillissement. Paris : Armand Colin.

Caradec, V. et Eve, M. (2002). Sociabilité et diffusion des technologies de la communication. Une étude de cas auprès de « jeunes retraités ». Réseaux, 5(115), 151-179.

Clément, S., Dubreuil, C. et Milanovic, F. (1999). Figures de la vieillesse et technologie de la vigilance. Réseaux, 17(96), 121-143.

Clément, S., Mantovani, J. et Membrado, M. (2004). Du bon voisinage aux solidarités des proximités. Dans P. Pitaud (dir.), Solitude et isolement des personnes âgées. L'environnement solidaire. (p.105-138). Toulouse : Erès.

Cornet, G. et Carré, M. (2008). Technologies pour le soin, l'autonomie et le lien social des personnes âgées : quoi de neuf ? Gérontologie et société, 3(126), 113-128. 
de Beauvoir, S. (1970), La Vieillesse. Paris : Éditions Gallimard.

de La Boétie, E. (2013). Discours de la servitude volontaire. Paris : Flammarion.

Delage, M. et Lejeune, A., (dir.) (2009). La résilience de la personne âgée : un concept novateur pour prendre en soin la dépendance et la maladie d'Alzheimer. Marseille : Solal.

Duvernay, D. (2011). Des liens intergénérationnels à la transmission transgénérationnelle: vers une co-création marque-consommateur. Communication et organisation, 40, 37-58.

Ennuyer, B. (2013). Les malentendus de l'« autonomie » et de la « dépendance » dans le champ de la vieillesse. Le sociographe, 6, 139-157.

Jani-Lebris, H. \& Luquet, V. (1997). La téléalarme en France. Un instrument de proximité au service de la personne dans son environnement, CLEIRPPA.

Fawkes, J. (2015). A Jungian conscience: Self-awareness for public relations practice. Public Relations Review, 41, 726-733.

Foucart, J. (2003). La vieillesse : une construction sociale. Pensée plurielle, 2(6), 7 18.

Gana, K., Alaphilippe, D. et Bailly, N. (2004). Positive illusions and mental and physical health in later life. Aging and Mental Health, 8(1), 58-64.

Granjon, F. (2012). Reconnaissance et usages d'internet. Une sociologie critique des pratiques de l'informatique connectée. Paris : Presses des Mines.

Guillemard, A.-M. (1978). À propos de la nouvelle représentation activiste de la vieillesse. Gérontologie, 28, 43-47.

Grille AGGIR (mai 2016), Repéré à :

http://www.cnsa.fr/documentation/guide_aggir_2008.pdf.

Henaff-Pineau, P.-C. (2012). Le sénior sportif, une nouvelle figure du bien vieillir ? Les Politiques sociales, 1 et 2, 101-112.

Honneth, A. (2000). La lutte pour la reconnaissance. Paris : Cerf

Hopflinger, F. (1995). From ageisme to gerontologism ? Emerging images of aging in gerontology. Hummel C, Lative d'Epinay, C. (dir.), Images of aging in Western societies. Genève : CIG, 91-98.

Hummel, C. (2002). Qu'en faut-il faire ? Réflexions sur la construction sociale des troisième et quatrième âges. Carnets de bord en sciences humaines, 3, 68-77. 
Humbert, C. (2008). Un vieil enfant. Le recours à la métaphore de l'enfance dans les récits d'accompagnants. Gérontologie et société, 4(127), 103-113.

Lagacé, M., Laplante, J., Davignon, A. (2011). Construction sociale du vieillir dans les médias écrits canadiens : de la lourdeur de la vulnérabilité à l'insoutenable légèreté de l'être. Organisation et Communication, 40, 87-102.

Lavoie, J.-P., Rose, D., Burns, V., Covanti, V. (2011). La gentrification de la PetitePatrie. Quelle place et quel pouvoir pour les aînés ? Diversité urbaine, 11(1), 5980.

Mac Cracken, G. (1987), Culture and Consumption among the elderly: three research objectives in an emerging field. Ageing and Society, 7, 203-224.

Mucchielli A. (2009), Dictionnaire des méthodes qualitatives en sciences humaines et sociales. Paris : Armand Colin.

Moschis, G.P. (1994), Consumer Behavior in Later Life: Multidisciplinary Contributions and Implications for Research. Journal of the Academy of Marketing Science, 22(3), 195-204.

Moscovici, S. (1984) The phenomenon of social representations. Dans Farr, R.M. et Moscovici, S. (dir.) Social representations. (p.3-70). London: Cambridge University Press.

Sen, A. (1999) Development as Freedom. Oxford : Oxford University Press.

Thomas, H. (2005). « Le «métier » de vieillard. Institutionnalisation de la dépendance et processus de désindividualisation dans la grande vieillesse ». Politix, 4(72), 33 55 .

Tréguer, J.-P. (2007). Le Sénior Marketing: Vendre et Communiquer Aux Générations de Plus de 50 Ans. Paris : Dunod.

Trincaz, J. (2015). Personne âgée : quelles représentations sociales ? Hier et aujourd'hui. Expertise collective INSERM, Activité physique et prévention des chutes chez les personnes âgées, 467-477.

Vigouroux-Zugasti, E. (2014). Quel regard sur les «vieux». Approche communicationnelle du lien social chez les Boomers in bloom. Communication et organisation, 45, 261-270.

Viriot Durandal, J.-P. (2012). «Le « pouvoir gris » du lobbying au pouvoir sur soi ». Gérontologie et société, 4(143), 23-38. 